\title{
Ligand-receptor interactions implicated in pathogenesis
}

The spondyloarthritis (SpA)-associated HLA variant $\mathrm{HLA} \mathrm{B}^{\star} 2705$ forms more heavy chain ligands and binds more strongly to killer cell immunoglobulinlike receptor 3DL2 (KIR3DL2) than the non-SpA-associated HLA-B ${ }^{\star} 2709$ variant. "Our results strongly implicate KIR3DL2-B27 interactions in SpA disease pathogenesis," summarizes senior investigator Simon Kollnberger.

"HLA-B27 has always been regarded as the key to understanding the pathogenesis," explains Professor Yu, an expert in the immunopathogenesis of SpA from the University College of Los Angeles, who was not involved in the study. "Every paradigm about how HLA-B27 causes SpA has to undergo the gauntlet of explaining why the disease does not occur in the HLA-B ${ }^{\star} 2709$ subtype individuals in Sardinia," he adds.

The researchers hypothesized that the differential association of HLA-B ${ }^{\star} 2705$ and HLA-B ${ }^{\star} 2709$ with SpA might result from differences in their formation of free heavy chain (FHC) ligands for KIR3DL2-a transmembrane protein expressed by natural killer (NK) cells and some T-cell types that can induce a survival signal when bound to ligand. In agreement with this theory, antibody staining and biochemical analysis detected more heavy chain dimers and FHC forms on the surface of

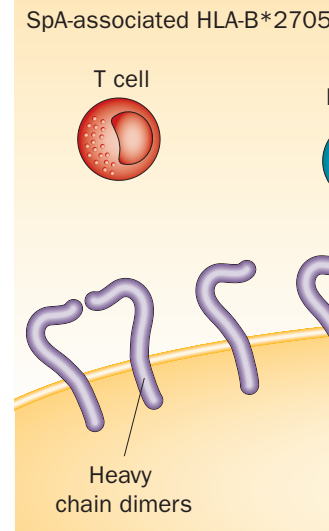

cells expressing HLA-B ${ }^{\star} 2705$ than those expressing HLA-B ${ }^{\star} 2709$. Accordingly, reporter-binding assays revealed greater T-cell stimulation by the HLA-B ${ }^{\star} 2705$ cells, which also increased NK cell survival to a larger extent than the HLA$B^{\star} 2709$ cells. Finally, the proportion of peripheral blood KIR3DL2-expressing $\mathrm{NK}$ and $\mathrm{CD}^{+}{ }^{+} \mathrm{T}$ cells was greater in patients with HLA-B ${ }^{\star} 2705$-positive SpA than in control individuals.

The investigators propose that differences in the formation of free heavy chains and heavy chain dimers can induce SpA. "The results of this study partially support the hypothesis," says Yu, noting that a complete lack of FHC and FHC dimers on HLA-B ${ }^{\star} 2709$ cells would have been a 'perfect' result for the theory. It

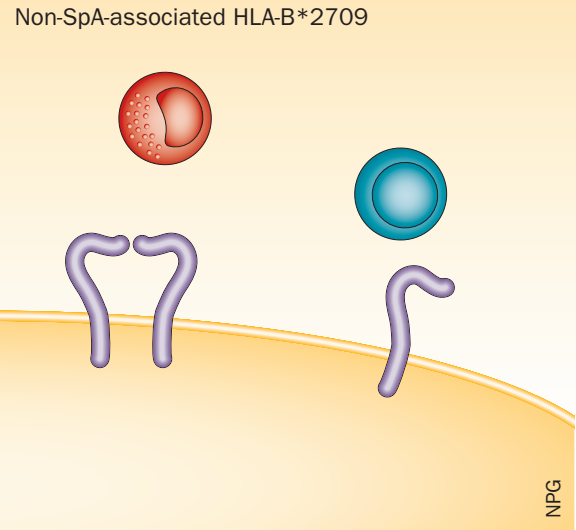

would also be strengthened, he adds, by comparison with individuals carrying the SpA-associated HLA-B ${ }^{\star} 2704$ variant.

Blocking the activity of aberrant HLA variants might provide new therapeutic directions in SpA. "Targeting KIR3DL2binding to B27 FHC could inhibit the expansion of pathogenic T-cell (and other leukocyte) subsets in SpA and lead to future therapeutics for treating arthritis," concludes Kollnberger.

Bryony Jones

Original article Cauli, A. et al. The arthritis-associated HLA$B * 27: 05$ allele forms more cell surface B27 dimer and free heavy chain ligands for KIR3DL2 than HLA-B*27:09. Rheumatology doi:10.1093/rheumatology/ket219 